\title{
Modification in Design of Monowheel
}

\author{
${ }^{1 .}$ Harish Kumar, ${ }^{2 .} \mathrm{K}$ B Anand, ${ }^{3 .}$ Paras Jain \\ 1. Assistant Professor, TMU, Moradabad, U.P, India \\ 2. Assistant Professor, TMU, Moradabad, U.P, India \\ 3. B.Tech.4th yr. Student, TMU, Moradabad, U.P., India,
}

\begin{abstract}
An engine-propelled monowheel vehicle comprises two wheels, close together, that circumscribe the remainder of the vehicle. When the vehicle is moving forward, the closely spaced wheels act as a single wheel, and the vehicle turns by leaning the wheels. A single propulsion system provides a drive torque that is shared by the two wheels. A separate steering torque, provided by a steering motor, is added to one wheel while being subtracted from the other wheel, enabling the wheels to rotate in opposite directions for turning the vehicle at zero forward velocity. A flywheel in the vehicle spins at a high rate around a spin axis, wherein the spin axis is rotatable with respect to the vehicle's frame. The axis angle and flywheel spin speed are continually adjustable to generate torques for automatic balancing.
\end{abstract}

\section{INTRODUCTION}

A monowheel is a one-wheeled single-track vehicle similar to a unicycle. Instead of sitting above the wheel as in a unicycle, the rider sits either within the wheel or next to it. The wheel is a ring, usually driven by smaller wheels pressing against its inner rim. Most are single-passenger vehicles, though multi-passenger models have been built. Hand- cranked and pedal-powered monowheel were patented and built in the late 19th century; most built in the 20 th century have been motorized. Some modern builders refer to these vehicles as monocycles, though that term is also sometimes used to describe motorized unicycles. Today, monowheel are generally built and used for fun and entertainment purposes, though from the 1860s through to the 1930s, they were proposed for use as serious transportation.

The world speed record for a motorized monowheel is $98.464 \mathrm{~km} / \mathrm{h}$ (61.18 mph).

\section{DESIGN}

\section{Design of Mono Wheel}

Then mono wheel with an effective power transmission system has to be designed such that it can be handled and controlled by a single person even in the rest position. The main and basic constraints that are being taken into consideration are

1. Height of the person riding the vehicle

2. Maximum weights that the vehicle can withstand

3. Power Transmissions

\section{Height of the person riding the vehicle}

According to the survey conducted an independent research organization average height of the person in India is $1.67 \mathrm{~m}$. This includes the people from both the genders .So the vehicle has been designed for people whose height lies in the range of $1.6 \mathrm{~m}(5,2$ ') $-1.8 \mathrm{~m}(5,9$ '). The average length of a leg person is $1.0 \mathrm{~m}$ and the height variation is mainly due to the variation in the growth of upper part of the body. So the vehicle accounts to a mean diameter of $1.4 \mathrm{~m}$ of which $0.6 \mathrm{~m}$ consists of the transmission system and the lower part of the body i.e. the legs and the lower abdomen manage this area of the vehicle and the upper part of the body occupy the rest of the area. The key advantage of this design is that the rider can easily balance the entire vehicle with the help of his legs and the person can feel comfortable while riding the vehicle.

\section{Maximum Weight the vehicle can withstand}

The vehicle that is fabricated should be able have a specification of the weight that it can bear. This is the Maximum weight bearing capacity of the vehicle. So, we have taken into consideration that the maximum weight of the rider to be $80 \mathrm{Kgs}$.

\section{STATEMENT OF THE PROBLEM OR HYPOTHESIS}

1. The monowheels are very unstable in low speed but very stable in speeds over $30 \mathrm{~km}$.

2. As we are talking about a stability of the vehicle we have to ensure that Centre of gravity and Centre of mass stays low so rider don't feel unstable when taking turn or vehicle doesn't falls to one direction.

3. As for braking system instead of using hard braking we have to use soft braking.

4.

\section{PROGRESS}

We have completed design phase 1 which includes main design of monowheel and some parts design. Case study have to be done and proper analysis needed which will take around I week.
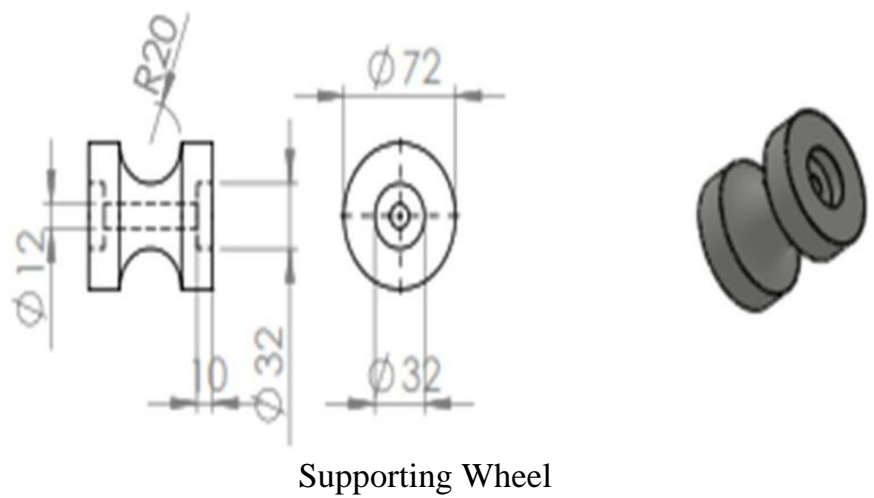

Supporting Wheel 


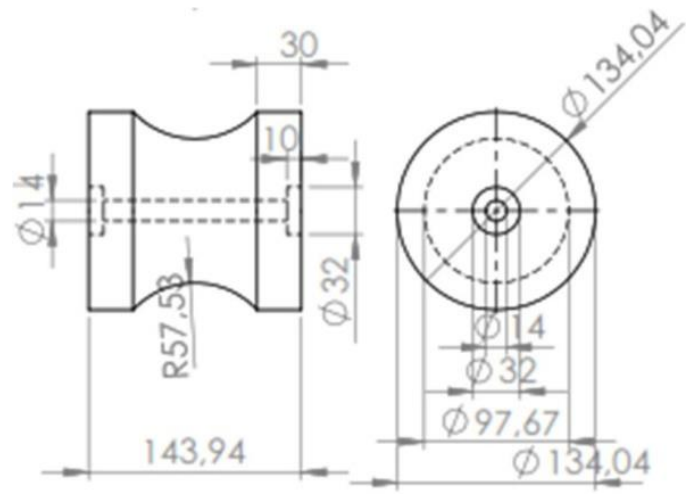

Driven Wheel

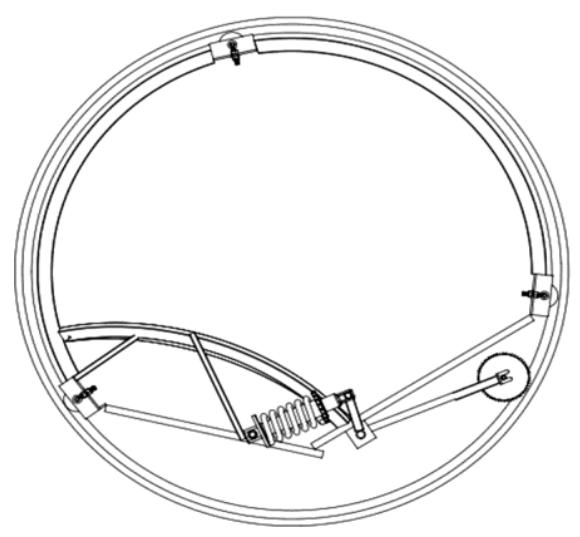

Basic Design Of Monowheel
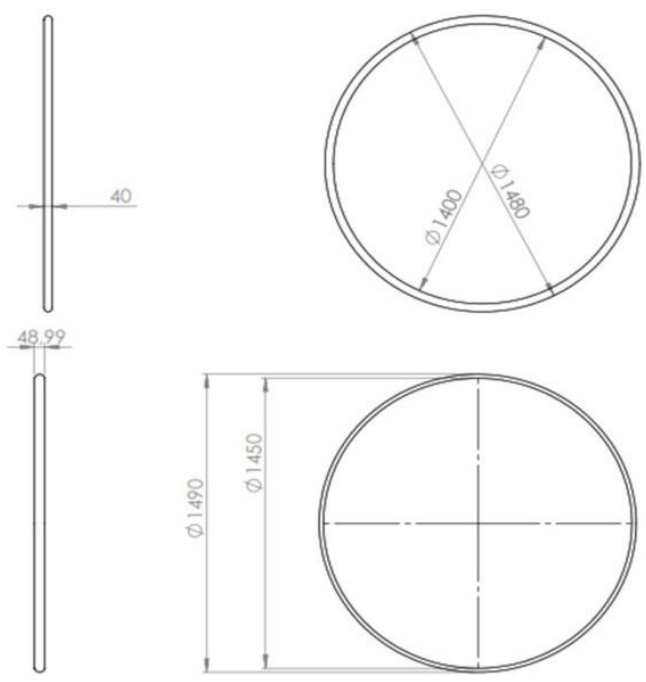

Main Wheel And Tyre Dimension

What is claimed is:

1. A vehicle comprising: a wheel having a diameter, an outer periphery of the whel for contacting a surface for moving relative to the surface; a support structure circumscribed by the wheel, wherein the wheel rotates about the support structure; a propulsion system circumscribed by the wheel and connected to the support structure, the propulsion system being coupled to the wheel for rotating the wheel around the propulsion system, thus propelling the wheel along the surface; and an automatic balancing system circumscribed by the wheel and connected to the support structure, the balancing system comprising: a flywheel that is controllable to spin around a flywheel spin axis, the flywheel spin axis being connected to the support structure by a pivot that is rotatable to orient the flywheel in different directions, whereby changing a direction of the flywheel spin axis places a torque on the support structure; and a control system configured to receive signals from one or more sensors in the vehicle providing balancing a propulsion motor; a transmission system coupled to the propulsion motor, the transmission system providing a drive torque; a differential gear assembly coupled to the transmission system and to the first wheel and the second wheel, the differential gear assembly splitting the drive torque between the first wheel and the second wheel while allowing the first wheel and the second wheel to rotate at different speeds; and a steering assembly coupled to the differential gear assembly, the steering assembly comprising a steering motor, the steering assembly being configured to supply a steering torque to the drive torque, where the steering torque is oppositely applied to the first wheel and second wheel to control relative rotations of the first wheel and the second wheel, enabling the first wheel and the second wheel to rotate in opposite directions to turn the vehicle.

2. The vehicle of claim $\mathbf{1}$ wherein the wheel is a first wheel, the vehicle further comprising a second wheel, wherein the support structure, propulsion system, and balancing system are circumscribed by the first wheel and the second wheel, wherein the first wheel and the second wheel are coupled to the propulsion system such that the first wheel and the second

3. The vehicle of claim $\mathbf{1}$ wherein the propulsion system comprises a propulsion motor and transmission coupling the propulsion motor to the wheel.

4. The vehicle of claim $\mathbf{1}$ wherein the control system orients the flywheel in different directions to steer the vehicle.

5. The vehicle of claim 1 wherein the flywheel is a first flywheel, the vehicle further comprising a second flywheel that is controllable to spin around its associated flywheel spin axis, the associated flywheel spin axis being connected to the second wheel are coaxial and the wheels are separated by at most $20 \%$ of one wheel diameter.

6. The vehicle of claim $\mathbf{5}$ wherein the second flywheel is configured to cancel any unwanted torque generated by the first flywheel.

7. The vehicle of claim $\mathbf{1}$ wherein the control system comprises a flywheel orientation motor coupled to the 
flywheel spin axis to change the direction of the flywheel spin axis.

8. The vehicle of claim $\mathbf{1}$ wherein the control system comprises a braking structure that decelerates rotation of the flywheel to provide a torque on the support structure for changing a tilt of the vehicle.

9. The vehicle of claim $\mathbf{1}$ wherein the flywheel comprises a motor that rotates about the flywheel spin axis, the control system comprising a power system coupled to the motor, the control system being configured to supply different drive signals to the motor to vary a rotational speed of the motor to adjust the attitude of the vehicle.

10. The vehicle of claim $\mathbf{1}$ wherein the wheel is a first wheel, the vehicle further comprising a second wheel, wherein the support structure, propulsion system, and balancing system are circumscribed by the first wheel and the second wheel, wherein the first wheel and the second wheel are coupled to the propulsion system such that the first wheel and the second wheel may rotate at different speeds, and wherein the propulsion system comprises: the first wheel for contacting a first portion of a surface; a second wheel having a diameter equal to the first diameter, an outer periphery of the second wheel contacting a second portion of the surface; a support structure circumscribed by the first wheel and the second wheel, wherein the first wheel and the second wheel rotate about the support structure; a propulsion system circumscribed by the first wheel and the second wheel and connected to the support structure, the propulsion system being coupled to the first wheel and the second wheel for rotating the first wheel and the second wheel around the propulsion system in a same direction to propel the vehicle in a forward direction, the propulsion system providing a drive torque to the first wheel and the second wheel; and a steering assembly coupled to the first wheel and the second wheel, the steering assembly comprising a steering motor, the steering assembly being configured to supply a steering torque to the drive torque, where the steering torque is oppositely applied to the first wheel and second wheel to control relative rotations of the first wheel and the second wheel, enabling the first wheel and the second wheel to rotate in opposite directions to turn the vehicle when the first wheel and the second wheel are both in contact with the surface.

11. The vehicle of claim $\mathbf{1}$ wherein the vehicle has roll, pitch, and yaw axes, and wherein the automatic balancing system provides torques about the roll, pitch, and yaw axes. Information, the control system being coupled to the 20 pivot to orient the flywheel in different directions to adjust an attitude of the vehicle for maintaining balance of the vehicle.

12. The vehicle of claim $\mathbf{1}$ wherein the one or more sensors comprise a plurality of attitude sensors for determining orientation of the vehicle.

13. The vehicle of claim $\mathbf{1}$ wherein a center of mass of the vehicle is located below an axis of rotation of the wheel.

14. The vehicle of claim $\mathbf{1}$ wherein the wheel is a first wheel, the vehicle further comprising a second wheel, wherein the support structure, propulsion system, and balancing system are circumscribed by the first wheel and the second wheel, wherein axes of rotation of the first wheel and the wheel may rotate at different speeds.

15. The vehicle of claim $\mathbf{1}$ wherein the wheel comprises a tire, wherein a profile of the tire perpendicular to the tire's diameter is a curve, the curve being convex away from the center of mass of the vehicle such that the vehicle's center of mass is at a minimum gravitational potential energy state at zero lean of the vehicle.

16. A vehicle comprising: a first wheel having a first diameter, an outer periphery of support structure by an associated pivot that is rotatable to orient the second flywheel in different directions, whereby changing a direction of the associated flywheel spin axis places a torque on the support structure.

17. The vehicle of claim $\mathbf{1 6}$ wherein the drive torque is substantially zero when the steering assembly rotates the first wheel and the second wheel in opposite directions.

18. The vehicle of claim $\mathbf{1 6}$ wherein the propulsion system comprises: a propulsion motor; a transmission system coupled to the propulsion motor, the transmission system providing the drive torque; and a differential gear assembly coupled to the transmission system and to the first wheel and the second wheel, the differential gear assembly splitting the drive torque between the first wheel and the second wheel thereby allowing the first wheel and the second wheel to rotate at different speeds, wherein the steering assembly is coupled to the differential gear assembly.

19. The vehicle of claim $\mathbf{1 6}$ wherein the propulsion system comprises: a differential gear assembly, the differential gear assembly Comprising a first differential gear coupled to the first wheel and a second differential gear coupled to the second wheel, the differential gear assembly splitting the drive torque between the first wheel and the second wheel thereby allowing the first wheel and the second wheel to rotate at different speeds; wherein the steering assembly comprises a steering gear engaging the first differential gear and the second differential gear, the steering gear being coupled to the steering motor for rotating the steering gear to cause the first differential gear and the second differential gear to rotate in opposite directions.

20. The vehicle of claim $\mathbf{1 9}$ wherein the steering gear is a pinion gear that engages teeth of the first differential gear and the second differential gear, wherein the pinion gear rotates about its axis of rotation to rotate the first differential gear and the second differential gear in opposite directions.

21. The vehicle of claim $\mathbf{2 0}$ wherein the pinion gear also 
rotates in a plane parallel to first differential gear and the second differential gear when the vehicle is moving in a forward direction.

22. A method for automatically balancing a vehicle, the vehicle being of the type comprising a wheel, the wheel having a diameter, an outer periphery of the wheel for contacting a surface for moving relative to the surface, the vehicle further including a support structure circumscribed by the wheel, wherein the wheel rotates about the support structure, the vehicle further including a propulsion system circumscribed by the wheel and connected to the support structure, the propulsion system being coupled to the wheel for rotating the wheel around the propulsion system, thus propelling the wheel along the surface, the vehicle requiring an automatic balancing system to be in a balanced state, the method comprising: providing a flywheel, circumscribed by the wheel, that spins around a flywheel spin axis, the flywheel spin axis being connected to the support structure by a pivot; sensing an imbalance in the vehicle; and in response to sensing the imbalance, automatically rotating the flywheel around the pivot to change a direction of the flywheel spin axis so as to place a torque on the support structure to adjust an attitude of the vehicle.

23. The method of claim $\mathbf{2 2}$ further comprising changing a rotational speed of the flywheel around the spin axis thereby placing a torque on the support structure to adjust an attitude of the vehicle for maintaining balance of the vehicle.

24. The method of claim $\mathbf{2 3}$ wherein changing a rotational speed of the $\mathrm{f} l \mathrm{y} \mathrm{w}$ h e e 1 around $\mathrm{t} h \mathrm{e}$ spin axis comprises roll axis being parallel to the surface in contact with the wheel and in the direction of movement of the vehicle.

25. The method of claim $\mathbf{2 2}$ wherein rotating the flywheel around the pivot corrects for imbalance about a roll axis, the direction to propel the vehicle in a forward direction, the propulsion system providing a drive torque to the first wheel and the second wheel; the method comprising: providing a steering assembly coupled to the first wheel and the second wheel, the steering assembly comprising a steering motor, separate from the propulsion system; and actuating the steering motor to supply a steering torque to the drive torque, where the steering torque is oppositely applied to the first wheel and second wheel to control relative rotations of the first wheel and the second wheel enabling the first wheel and the second wheel to rotate in opposite directions to tum the vehicle when the first wheel and the second wheel are both in contact with the surface.

26. The method of claim $\mathbf{2 2}$ further comprising rotating the flywheel around the pivot, so as to place a torque on the support structure, causing the vehicle to lean in order to steer the vehicle.

27. The method of claim 22 further comprising changing a rotational speed of the flywheel around the spin axis, so as to place a torque on the support structure, causing the vehicle to lean in order to steer the vehicle.

28. The method of claim $\mathbf{2 2}$ further comprising performing at least one of rotating the flywheel around the pivot and changing a rotational speed of the flywheel around the spin axis for righting the vehicle from a horizontal position.

29. The method of claim $\mathbf{2 8}$ wherein the vehicle has a roll axis, wherein righting the vehicle from a horizontal position comprises: establishing a first angular momentum of the flywheel oriented substantially parallel to the roll axis; and changing the angular momentum to cause a reactionary torque, righting the vehicle.

30. The method of claim $\mathbf{2 9}$ wherein changing the angular momentum comprises braking the flywheel.

31. The method of claim $\mathbf{3 0}$ wherein braking the flywheel is sufficiently rapid to cause the vehicle to experience an aerial phase as it is righted, where the vehicle is temporarily not in contact with the surface.

32. The method of claim $\mathbf{2 2}$ wherein sensing an imbalance in the vehicle and rotating the flywheel around the pivot comprises: determining a present attitude vector of the vehicle; determining a net torque vector that, if provided to the vehicle, would provide an acceleration about roll, pitch, and yaw axes of the vehicle; determining control signals needed to change an angular momentum of the flywheel; and applying said control signals to produce a desired net torque vector.

33. The method of claim 22 wherein sensing an imbalance in the vehicle and rotating the flywheel around the pivot comprises: determining a present attitude vector of the vehicle and a time rate of change of the attitude vector; determining a net torque vector that, if provided to the vehicle, would provide an acceleration about roll, pitch, and yaw axes of the vehicle; determining control signals needed to change an angular momentum of the flywheel; and applying said control signals to produce a desired net torque vector.

34. A method for steering a vehicle, the vehicle being of the type having a first wheel having a first diameter, an outer periphery of the first wheel contacting a first portion of a surface, the vehicle also including a second wheel having a diameter equal to the first diameter, an outer periphery of the second wheel contacting a second portion of the surface, the vehicle also including a support structure circumscribed by the first wheel and the second wheel, wherein the first wheel and the second wheel rotate about the support structure, the vehicle also including a propulsion system circumscribed by the first wheel and the second wheel and connected to the support structure, the propulsion system being coupled to the first wheel and the second wheel for rotating the first wheel and the second wheel around the propulsion system in a same mechanically braking the flywheel.

35. The method of claim $\mathbf{3 4}$ wherein the propulsion system comprises a differential gear assembly, the differential gear assembly comprising a first 
differential gear coupled to the first wheel and a second differential gear coupled to the second wheel, the differential gear assembly splitting the drive torque between the first wheel and the second wheel while a second output shaft coupled to a second wheel for rotating the second wheel, the second output shaft being coupled to the second differential gear such that rotation of the second differential gear rotates the second wheel a differential gear coupling engaging both the first differential gear and the second differential gear so as to substantially evenly split a first input torque from the first rotating element between the first output shaft and the second output shaft; and a second rotating element driven by a second drive system, the second rotating element engaging the first differential gear and the second differential gear, wherein rotating the second rotating element changes a torque applied to the first differential gear by a first magnitude and changes a torque applied to the second differential gear by a second magnitude opposite to the first magnitude.

36. The method of claim $\mathbf{3 5}$ wherein the steering gear is a pinon gear that engages teeth of the first differential gear and the second differential gear, wherein the pinion gear rotates about its axis of rotation to rotate the first differential gear and the second differential gear in opposite directions.

37. The method of claim $\mathbf{3 6}$ wherein the pinion gear also rotates in a plane parallel to the first differential gear and the second differential gear when the vehicle is moving in a forward direction.

38. A differential gear assembly comprising: a first rotating element driven by a first drive system; a first differential gear coupled to the first rotating element.

39. The differential gear assembly of claim 38 wherein the second rotating element comprises a pinion gear that engages teeth of the first differential gear and teeth of the second allowing the first wheel and the second wheel to rotate at different speeds, and wherein the steering assembly compasses a steering gear engaging the first differential gear and the second differential gear, the steering gear being coupled to the steering motor, the method further comprising: differential gear, wherein the pinion gear rotates about its axis of rotation to change the torque applied to the first differential gear by the first magnitude and change the torque applied to the second differential gear by the second magnitude opposite to the first magnitude actuating the steering motor for rotating the steering gear to cause the first differential gear and the second differential gear to rotate in opposite directions.

40. The differential gear assembly of claim 39 wherein the teeth of the first differential gear and the teeth of the second differential gear face each other, and wherein the pinion gear is sandwiched between the first differential gear and the second differential gear.

41. The differential gear assembly of claim 40 wherein the pinion gear has a shaft that extends beyond a periphery of the first differential gear and the second differential gear, wherein the shaft is engaged and rotated by the second rotating element.

42. The differential gear assembly of claim 41 wherein the pinion gear, by simultaneously engaging the teeth of the first differential gear and the teeth of the second differential gear, revolves around an axis of rotation of the first differential gear and the second differential gear while also allowing the pin wherein rotation of the first rotating element rotates the first differential gear; a first output shaft coupled to a first wheel for rotating the first wheel, the first output shaft being coupled to the first differential gear such that rotation of the first differential gear rotates the first wheel; a second differential gear coupled to the first differential gear so as to allow the second differential gear and the first differential gear to rotate at different speeds; an gear to independently rotate about its axis of rotation.

43. The differential gear assembly of claim 42 wherein the second rotating element comprises a ring gear that circumscribes the first differential gear and the second differential gear, the ring gear engaging teeth formed around the pinion gear shaft, wherein rotation of the ring gear by the second drive system rotates the pinion gear about its axis of rotation.

\section{WORKING METHODOLOGY}

We have to start our structure by making a big, metallic wheel with $140 \mathrm{~cm}$ diameter using a tube of $40 \mathrm{~mm}$ diameter. Then, we have to line the metallic wheel with a rubber in order to achieve having the benefits that all the tyres have on the street. Also, due to the fact that the wheel is big in size, we took 4 tyres from small motorbikes and we cut they as well as we glued the one with the other in order to form a tyre. Afterwards, we made 3 wheels with a bearing using Teflon PTFE and on which the big wheel rolls. These three wheels were attached with the inside part of the wheel on a framework. On this framework, another machine 110cc 4 stroke was placed. This machine using a chain, enables a rubber wheel to move so this wheel enables the big wheel to move as well. Therefore, the rider sits inside the wheel.

[1] Patent No. US 8,219,308 B2

\section{REFERENCE}

[2] "Improvement in velocipede: US 92528 A". Google India. Retrieved July 21, 2016.

[3] Goddard, J. T. (1869). The velocipede: its history, varieties, and practice. University of Princeton: Hurd and Houghton. pp. 76-78.

[4] US 325548, J.O. Lose, "One wheeled vehicle", issued 1 September 1885

[5] "Guinness World Record for bearded woman Harnaam Kaur". BBC News. Retrieved 8 September 2016. 